\title{
DYNAMICS OF THE MIMAS-TETHYS SYSTEM
}

\author{
SYLVAIN CHAMPENOIS \\ Laboratoire d'Astronomie, 1 Impasse de l'Observatoire, 59000 LILLE, \\ E-mail: Sylvain.Champenois@bdl.fr
}

\begin{abstract}
The role of the $200 \mathrm{yr}$-long period found recently in the mean longitude of Mimas (Vienne and Duriez 1992) is investigated through numerical integrations. It is shown that it has a deciding effect on the descriptions of the resonance motion of the Mimas-Tethys system, as considered up to now. As a result, Mimas's inclination before capture may have been higher (up to $0.7^{\circ}$ ) or lower (down to $0.03^{\circ}$ ) than the value previously considered $\left(0.42^{\circ}\right)$. Also, Tethys's eccentricity on capture may have been quite higher $(\approx 0.008$ versus 0 ). Moreover, the probability of capture is found to be very sensitive to Tethys's eccentricity, and possibly much higher (up to 1 ) than the value considered before (0.04).
\end{abstract}

\section{Introduction}

Mimas and Tethys are the first and third satellite of saturn. We use the following notations: $a, n, e, i, \gamma, \varpi, \Omega, \lambda$ and $m$ are Mimas's orbital semimajor axis, mean motion, eccentricity, inclination, sine of semi-inclination, longitude of periapse, longitude of ascending node, mean longitude and mass (in units of Saturn's mass), respectively; the corresponding primed quantities refer to Tethys. These two satellites are connected by an inclination-type resonance: the argument $\varphi=2 \lambda-4 \lambda^{\prime}+\Omega+\Omega^{\prime}$ slowly oscillates around zero with a great amplitude $\left(95^{\circ}\right.$ with a period of 70 years). Since the argument $\varphi$ is present in terms factored by $i i^{\prime}$ in the expansion of the disturbing potential, we shall call this resonance the $i i^{\prime}$ resonance.

Up to now, the dynamics of this resonance had been studied assuming that, close to the resonance, the period of the resonant argument is much longer than the periods of all the other arguments present in the right-hand side of Lagrange's equations. This allows to average out all the non-resonnant arguments in those equations. To this averaged equations were added tidal effects on the satellites' mean motions, due to dissipation in Saturn.

According to this analysis, $i$ and $i^{\prime}$ are constant before capture, while the ratio $\alpha=a / a^{\prime}$ increases under tidal effects, causing the system to come closer and closer to the resonance. Once the system is captured, $\alpha$ starts oscillating around the value corresponding to strict resonance, while $i$ and $i^{\prime}$ start increasing. Tethys being 17 times more massive than Mimas, its inclination has remained almost unchanged since capture up to the present time, $\left(\approx 1.0^{\circ}\right)$. On the other hand, Mimas's one has increased fourfold in the same time, from $0.4^{\circ}$ to $1.6^{\circ}$ (Allan 1969; Champenois and Vienne 1999a).

The recent discovery, by Vienne and Duriez (1992), of long-period terms in the mean longitude of Mimas, lead us to reconsider this classical analysis. As a matter of fact, we realized that we could not retain the only resonnant argument in the right-hand side of the equations of the Mimas-Tethys system, since the periods of 
some of the non-resonant arguments were not, close to the resonance, much lower than the period of the resonant argument.

\section{A crucial Low Frequency}

Among the long periods discovered, one of them, with period 200 years, is particularly interesting because of its relative closeness to the period of the resonant argument (which is currently 70 years, and was higher in the past). This period has its origin in the argument $\sigma=\frac{1}{2} \Omega-\frac{3}{2} \Omega^{\prime}+\varpi^{\prime}$, which appears in the right-hand side of Lagrange's equation through the following three main arguments :

$$
\begin{aligned}
\frac{3 \varphi}{2}+\sigma & =3 \lambda-6 \lambda^{\prime}+2 \Omega+\varpi^{\prime} \\
\frac{\varphi}{2}+\sigma & =\lambda-2 \lambda^{\prime}+\Omega-\Omega^{\prime}+\varpi^{\prime} \\
-\frac{\varphi}{2}+\sigma & =-\lambda+2 \lambda^{\prime}-2 \Omega^{\prime}+\varpi^{\prime}
\end{aligned}
$$

The terms of these arguments are factored by the respective quantities $i^{2} e^{\prime}, i i^{\prime} e^{\prime}$ and $i^{\prime 2} e^{\prime}$ in the expansion of the disturbing potential. The value of $e^{\prime}$ is very badly known. The TASS1.6 theory (Vienne and Duriez 1995) gives $e^{\prime}=0.000235$, but with such uncertainties that it lies actually in the interval $[0,0.001]$. Hence, we shall consider in what follows several possible current values. A certain thing, however, is that $e^{\prime}$ is very small at the present time. But this was not the same in the past, if we take into account its damping by the tides raised on Tethys by Saturn. Dermott et al. (1988) give for the time scale $\tau$ of the damping of Tethys' eccentricity the value $\tau=10^{8} \mathrm{yr}$. This is smaller than the age of the Mimas-Tethys resonance, which can be deduced from Allan's study to be $\approx 2.4 \tau$ (Champenois and Vienne 1999a). Therefore $e^{\prime}$ may have been sensibly higher on time of capture into the present resonance than at the present time (up to 0.01).

Hence, we can expect the presence of $j / k$ secondary resonances between the libration or circulation frequency $\omega$ of the primary $i i^{\prime}$ resonance, and the frequency $\dot{\sigma}$ (defined by the relation $j \omega+k \dot{\sigma}=0$ ), as well as the presence of chaotic zones due to the overlapping of these secondary resonances near the border of the $i i^{\prime}$ resonance.

\section{Numerical Integrations}

Averaged Lagrange's equations are considered: only the following four arguments, with periods of the order of $10^{2}$ years, are retained in the expansion of the disturbing potential: $\varphi, \frac{3}{2} \varphi+\sigma, \frac{1}{2} \varphi+\sigma$ and $-\frac{1}{2} \varphi+\sigma$. In particular, secular terms depending on longitudes of nodes or pericenters (like $e e^{\prime} \cos \left(\varpi-\varpi^{\prime}\right)$ ) are removed, because their periods are of the order of the year. Moreover, only the largest terms have been retained in the right-hand side of the averaged equations. Then the main terms coming from the oblateness of Saturn are added (up to $J_{2}^{3}$ ), as well as tidal terms 
on $n, n^{\prime}$ and $e^{\prime}$. Regular variables are considered. We integrated these equations backwards in time, for various initial values $e_{0}^{\prime}$ of $e^{\prime}$ (subscript 0 is for the present time). The initial conditions are taken from TASS1.6 for J2000.

$\dot{\sigma}$ was found to be only slowly varying since capture time (from $\approx 2 \pi / 185 \mathrm{yr}$ to $\approx 2 \pi / 200 \mathrm{yr}$ at the present time). Therefore, in order to locate secondary resonances in an $\left(\alpha, y=\sqrt{\gamma \gamma^{\prime}}\right)$ plane, we may consider it constant throughout that period. Using a pendulum model for the $i i^{\prime}$ resonance, we thus obtain the curves labelled $j / k$, shown on Fig 1 . The V-shaped zone on these figures is obtained from the same model. This is the libration zone of the $i i^{\prime}$ resonance: inside it, $\alpha$ oscillates around the value $\alpha_{r}=0.629308514$ corresponding to strict resonance, between two extreme values. During one period of these oscillations, $y$ increases in an imperceptible way. Only the borders of these librations are plotted on these figures.

Figure 1 shows, on the left, the results obtained for $e_{0}^{\prime}=0$ (1), 0.00235 (2) and 0.001 (3), the other initial conditions being taken from TASS1.6. We notice that $y$ on capture increases with $e_{0}^{\prime}$. But $i^{\prime}$ is found to be insensitive to $e_{0}^{\prime}$. Thus, $i$ on capture increases with $e_{0}^{\prime}$. We have: $0<e_{0}^{\prime}<0.001 \Rightarrow 0.4^{\circ}<i<0.7^{\circ}$ and $810^{-5}<e^{\prime}<0.008$ at capture time. On the right is shown a run also obtained for $e_{0}^{\prime}=0.001$, but with a slight change in Mimas's initial mean motion compared to the previous run (by $510^{-4} \mathrm{rad} \mathrm{yr}^{-1}$ : this remains within the range of uncertainties). This run reveals a capture in the $1 / 2$ secondary resonance: the system enters the primary resonance with $i=0.18^{\circ}$ and $e^{\prime}=0.008(1)$. After capture, there are large oscillations in the libration amplitude (2), followed by a jump to about $30^{\circ}$. Then the trajectory follows the adiabatic invariant theory (3; see Champenois and Vienne 1999a), until it gets trapped in the $1 / 2$ secondary resonance (4). After escaping from this secondary resonance, the libration amplitude decreases again smoothly up to the present time (5). Other runs also reveal the possibility for a capture in the $1 / 1$ secondary resonance.

\section{Surfaces of Section}

Surfaces of section are computed thanks to the following equation (Champenois and Vienne 1999a):

$$
\begin{aligned}
\ddot{\varphi}= & R_{0}\left(\sin (\varphi)+R_{1} \sin \left(\frac{3}{2} \varphi+\dot{\sigma} t+\sigma_{0}\right)\right. \\
& \left.+R_{2} \sin \left(\frac{1}{2} \varphi+\dot{\sigma} t+\sigma_{0}\right)+R_{3} \sin \left(-\frac{1}{2} \varphi+\dot{\sigma} t+\sigma_{0}\right)\right)
\end{aligned}
$$

where the coefficients $R_{i},(0 \leq i \leq 3)$ essentially depend on $e^{\prime}$ and $i$. If $i>0.1^{\circ}$, then they may also be viewed as parameters, and Eq. (1) becomes a periodically time-dependent one-degree-of-freedom equation, in which $\dot{\sigma}$ is fixed at the time at which we want to compute our surfaces of section.

Figure 2 shows the surfaces of section $(\varphi, \dot{\varphi})$ of the Mimas-Tethys system for trajectories 2 (left) and 3 (right), shown on Fig. 1, both at present time (up) and 

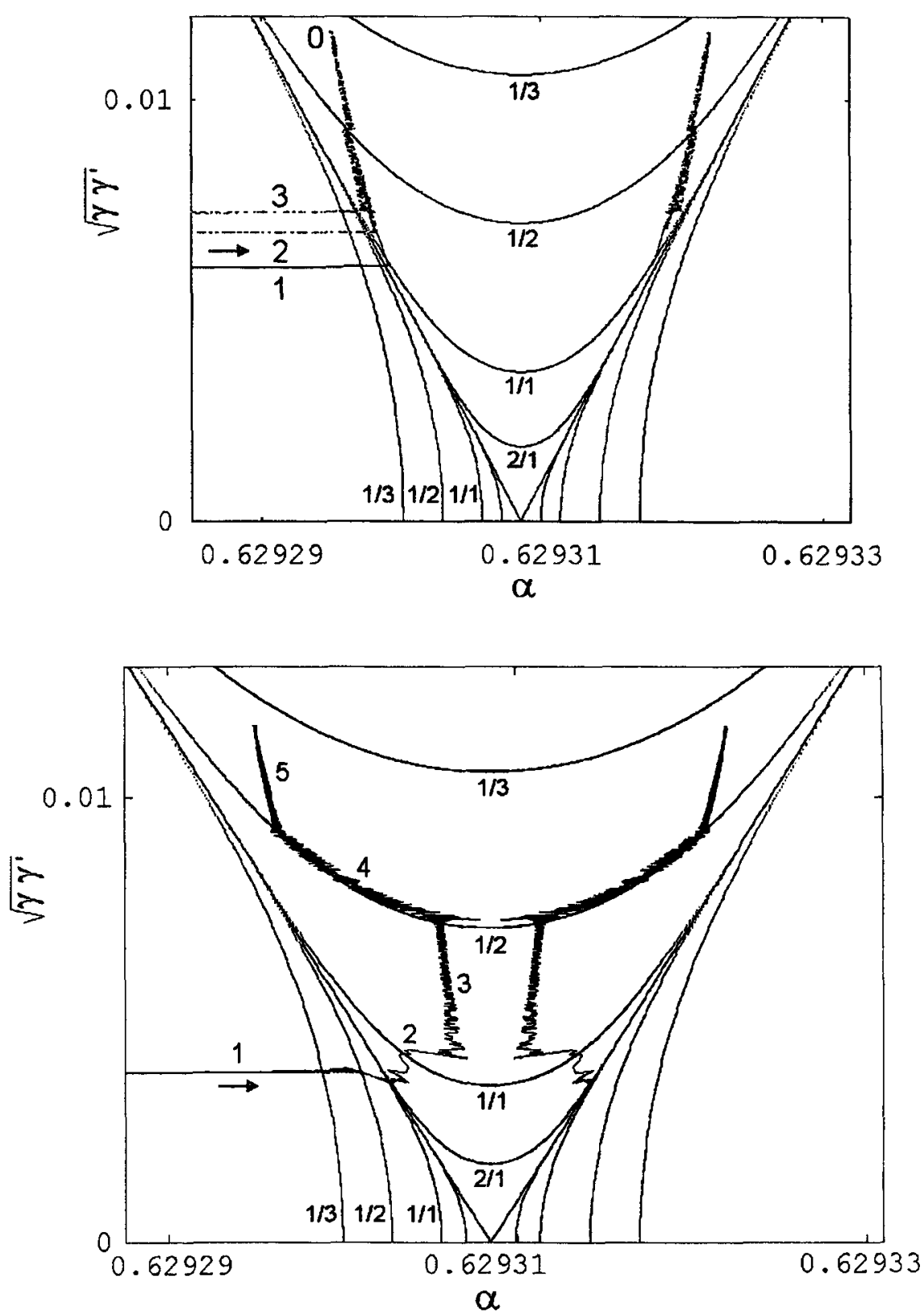

Fig. 1. Different possible trajectories for the past evolution of the Mimas-Tethys system. in the bottom panel is shown a possible capture in the $1 / 2$ secondary resonance. The arrow indicates the direction of evolution, and 0 is for the present time. The $j / k$ curves correspond to secondary resonances, the V-shaped zone to the primary libration zone. See text. 

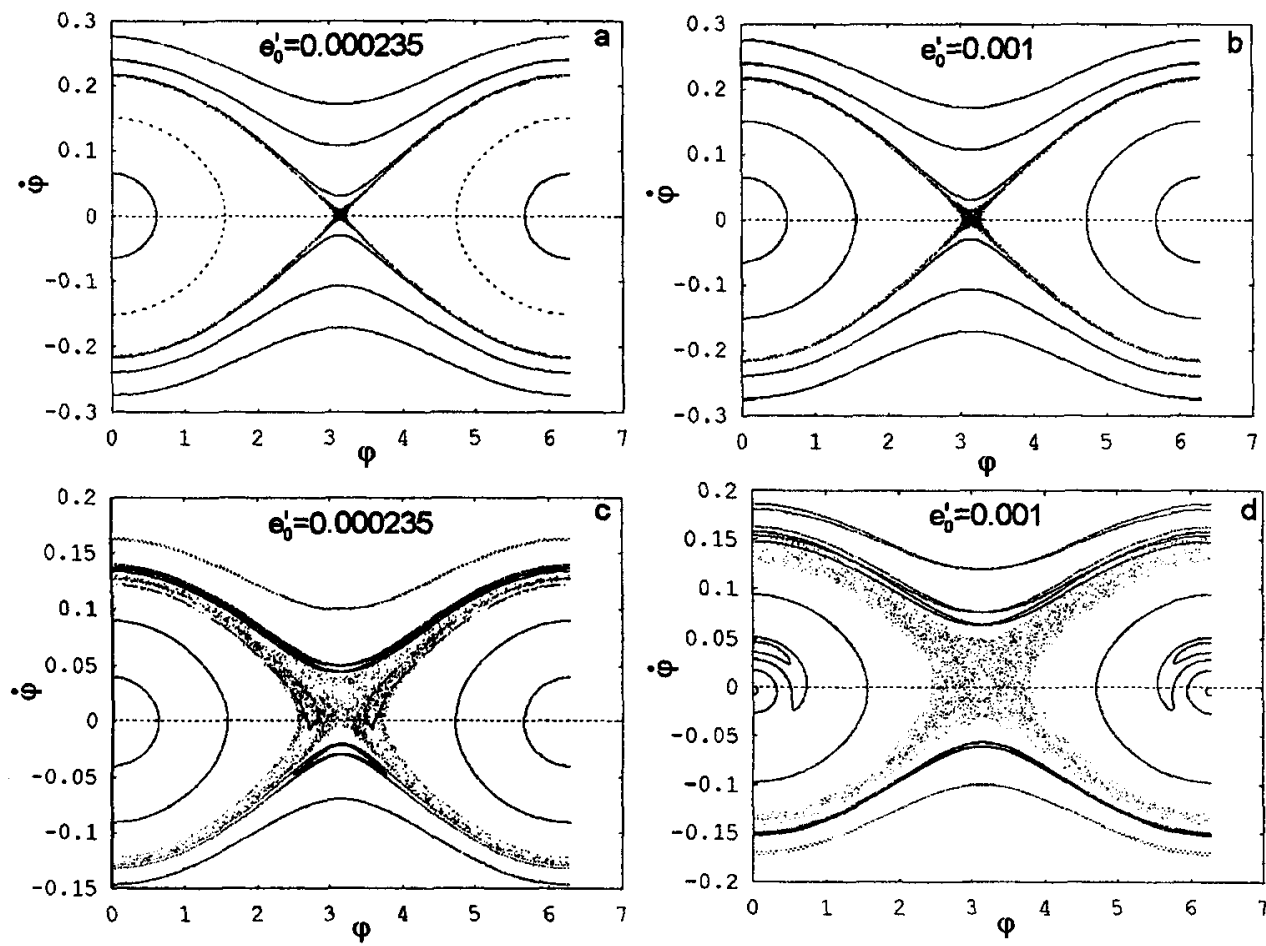

Fig. 2. Surfaces of section of the Mimas-Tethys system at present time (up) and capture time (down), for $e_{0}^{\prime}=0.000235$ (left) and $e_{0}^{\prime}=0.001$ (right), corresponding to trajectories 2 and 3 shown on Fig. 1. See text for comments.

capture time (down). This figure reveals narrow chaotic borders of the $i i^{\prime}$ resonance at present time, but large ones at capture time (when the eccentricity of Tethys is bigger), as well as islands associated to the $1 / 1$ and $1 / 2$ secondary resonances.

\section{Capture Probability}

To evaluate the probability of capture, Sinclair (1972) uses for the Mimas-Tethys resonance a pendulum model, whose "restoring force" increases with time. He then uses for the probability of capture the following formula: $P=(u / v)^{2}$, where $u$ is the value of $\varphi$ (above the unstable equilibrium point, near $\varphi=-\pi$ ) separating capture from escape, and $v$ is the value separating escape from direct circulation. He thus obtains $P=0.04$.

Taking now $\sigma$ into account, there is now one more choice in addition to capture and inverse circulation: the system may also librate and circulate in turn, in a chaotic way. However, tidal effects place bounds on it, urging the system to choose for good between capture and inverse circulation. If $m(\mathcal{C})$ is the measure of the set of values for which the system is captured for good, and $m(\mathcal{E})$ the measure of the 
set of values for which it escapes from resonance, we can then generalize Sinclair's formula in the following way (Champenois and Vienne 1998b):

$$
P=\left(\frac{m(\mathcal{C})}{m(\mathcal{C})+m(\mathcal{E})}\right)^{2}
$$

Applying formula (2), $P$ appears to vary strongly with $e^{\prime}$, increasing from $P=0.04$ for $e_{0}^{\prime}=0$ to $P=1$ for $e_{0}^{\prime}=0.0016$. The present uncertainty on $e_{0}^{\prime}$ therefore results in a big uncertainty on the capture probability into the present resonance. However, it seems likely to be higher than that determined by Sinclair.

\section{Conclusion}

We can see from this study that the consideration of the low frequency $\dot{\sigma}$ upsets the vision of the dynamics of the Mimas-Tethys system that we had so far. As a matter of fact, the system may have been trapped in a secondary resonance or may have behaved in a chaotic way on capture in the present $i i^{\prime}$ resonance. As a result, the inclination of Mimas may have been higher (up to $0.7^{\circ}$ ), or lower (down to $0.03^{\circ}$ ) than derived by Allan (1969). Moreover, the eccentricity of Tethys have been higher in the past (up to 0.008 ), and the probability of capture into the present resonance may be much higher (up to 1) then that found by Sinclair (1972).

\section{References}

Allan, R.R.:1969, Astron.J., 74, 497-506

Champenois, S. and Vienne, A.: 1999a, Icarus, accepted

Champenois, S. and Vienne, A.: 1999b, Cel. Mech. \& Dyn. Astro., submitted

Dermott, S.F., Malhotra, R. and Murray, C.D.:1988, Icarus, 76, 295-334

Sinclair, A.T.: 1972, Mon. Not. R. Astron. Soc., 160, 169-187

Vienne, A. and Duriez, L.: 1992, Astron. Astrophys., 257, 331-352

Vienne, A. and Duriez, L.: 1995, Astron. Astrophys., 297, 588-605 